\title{
Evaluación de la presencia de Adenocephalus pacificus en pescados de mayor consumo en el Perú en el periodo 2016-2017
}

\author{
Evaluation of the presence of Adenocephalus pacificus in fish of greater \\ consumption in Peru in the period 2016-2017
}

Pablo Londoñe-Bailón ${ }^{1,3}$, Roxana Céspedes-Chombo², Katherine Díaz Pereyra²

\section{Resumen}

El presente estudio tuvo como objetivo evaluar la prevalencia, abundancia e intensidad media de plerocercoides (PLs) de Adenocephalus pacificus en pescados de mayor importancia comercial de la costa peruana. Se muestrearon 28 especies de pescados y se analizaron 12017 especímenes. Se determinó la presencia de PLs de $A$. pacificus a partir de anchoveta (Engraulis ringens), lorna (Sciaena deliciosa), lisa (Mugil cephalus), caballa (Scomber japonicus), cabrilla (Paralabrax humeralis) y cabinza (Isacia conceptionis) con una prevalencia de $0.55,24.96,0.56,1.79,1.53$ y $3.94 \%$, respectivamente. Tres distintos tipos de escólex de PLs de A. pacificus fueron identificados como esférico, lanceolado y acorazonado, indistintamente del hospedero, zona y periodo de muestreo.

Palabras clave: Adenocephalus pacificus, plerocercoide, pescado comercial, descriptor estadístico

\section{Abstract}

The aim of this study was to evaluate the prevalence, abundance and average intensity of plerocercoids (PLs) of Adenocephalus pacificus in the most important commercial fish species from the Peruvian coast. A total of 28 species of fish were sampled and 12017 specimens were analysed. The presence of PLs of $A$. pacificus were determined from Peruvian anchovy (Engraulis ringens), lorna drum (Sciaena deliciosa), striped mullet

\footnotetext{
${ }^{1}$ Universidad Peruana Cayetano Heredia, Lima, Perú

${ }^{2}$ Laboratorio de Microbiología, Dirección de Investigación, Desarrollo, Innovación y Transferencia Tecnológica, Instituto Tecnológico de la Producción (ITP), Callao, Perú

${ }^{3}$ E-mail: pablo_londone@hotmail.com
}

Recibido: 1 de abril de 2019

Aceptado para publicación: 23 de enero de 2020

Publicado: 31 de marzo de 2020 
(Mugil cephalus), horse mackerel (Scomber japonicus), Peruvian rock sea bass (Paralabrax humeralis) and cabinza grunt (Isacia conceptionis) with a prevalence of $0.55,24.96,0.56,1.79,1.53$ y $3.94 \%$, respectively. Three different types of scolex of $A$. pacificus PLs, like as spherical, lanceolate, heart-shaped were identified, regardless of the host, area and sampling period.

Key words: Adenocephalus pacificus, plerocercoid, commercial fish, statistic descriptor

\section{INTRODUCCIÓN}

El Perú es uno de los países pesqueros más ricos del mundo y uno de los principales exportadores a nivel mundial (Shimokawa, 2013). La actividad pesquera comprende las actividades de extracción y transformación de recursos hidrobiológicos como peces, moluscos, crustáceos y otras especies para el consumo humano directo (enlatado, fresco o congelado) e indirecto (harina y aceite de pescado). Cinco especies de pescado (bonito [Sarda chiliensis], merluza [Merluccius gayi peruanus], caballa [S. japonicus], perico [Coryphaena hippurus] y lisa [M. cephalus]) presentaron el mayor desembarque para el consumo fresco durante $2016 \mathrm{y}$ 2017 (PRODUCE, 2016, 2017).

Los peces destinados al consumo se pueden encontrar infestados por especies parasitarias que pueden poner en riesgo la salud humana. La presencia de parásitos en productos de la pesca es inevitable y difícilmente controlada debido a que cohabitan en el mismo medio. Se han descrito numerosos parásitos en pescados de consumo, la mayoría solo deterioran la apariencia del pescado y devalúan su valor (Serrano-Martínez et al., 2017); sin embargo, existen otros tipos de parásitos de peces que son de importancia en la salud pública (Sánchez et al., 2016; Scholz y Kuchta, 2016).

Adenocephalus pacificus tiene importancia médica en el Perú y a nivel mundial. Ocasiona una enfermedad zoonótica y es el único agente causal de la difilobotriasis humana en el Perú (Tantaleán, 1993; Hernández-Orts et al., 2015; Kuchta et al., 2015; Sánchez et al., 2016; Scholz y Kuchta, 2016; Berger, 2017; Moore et al., 2017). Este parásito es endémico de las costas del Pacifico, por lo que la mayoría de los casos clíni$\cos (>99 \%)$ son reportados en el Perú; sin embargo, existen casos recientes reportados en Europa que corroboran su distribución mundial y la importancia de prestar mayor atención a esta zoonosis emergente (Kutcha et al., 2014).

En el Perú, la difilobotriasis es adquirida por el consumo de peces marinos, portadores de larvas de cestodos de la familia Diphyllobothridae, crudos o insuficientemente cocidos en forma de «cebiche», «tiradito» o «chinguirito» (Tantaleán, 1993; Kuchta et al., 2015; Scholz y Kuchta, 2016). Diversos estudios demuestran la presencia de este cestodo en peces de interés comercial en el país (Chero et al., 2014a,b,c, 2016; SerranoMartínez et al., 2017), por lo que es importante mantener el constante control de calidad e inspección sanitaria de peces de consumo (Scholz y Kuchta, 2016).

Los principales factores de riesgo que incrementan la expansión mundial de los parásitos (incluido A. pacificus) incluyen las importaciones de pescado refrigerado o insuficientemente congelado, la alta demanda del consumo de pescado crudo o poco cocido y los viajes y migraciones de personas con infecciones parasitarias a otros países (Kutcha et al., 2015). El objetivo del presente estudio 
Cuadro 1. Coordenadas geográficas de las 11 zonas de muestreo

\begin{tabular}{ccccc}
\hline $\mathrm{N}^{\circ}$ & Región & Provincia & \multicolumn{2}{c}{ Coordenadas } \\
\hline 1 & Callao & Callao & $12^{\circ} 02^{\prime} 49^{\prime \prime} \mathrm{S}$ & $77^{\circ} 08^{\prime} 34^{\prime \prime} \mathrm{O}$ \\
2 & Lima & Pucusana & $12^{\circ} 25^{\prime} 00^{\prime \prime} \mathrm{S}$ & $76^{\circ} 47^{\prime} 00^{\prime \prime O}$ \\
3 & Lambayeque & Chiclayo & $06^{\circ} 54^{\prime} 39^{\prime \prime} \mathrm{S}$ & $79^{\circ} 51^{\prime} 47^{\prime \prime} \mathrm{O}$ \\
4 & Ancash & Chimbote & $09^{\circ} 04^{\prime} 32^{\prime \prime} \mathrm{S}$ & $78^{\circ} 36^{\prime} 33^{\prime \prime} \mathrm{O}$ \\
5 & Ica & Pisco & $13^{\circ} 48^{\prime} 10^{\prime \prime} \mathrm{S}$ & $76^{\circ} 17^{\prime} 31^{\prime \prime O}$ \\
6 & Lima & Chorrillos & $12^{\circ} 09^{\prime} 52^{\prime \prime} \mathrm{S}$ & $77^{\circ} 01^{\prime} 48^{\prime \prime} \mathrm{O}$ \\
7 & Lima & Ancón & $11^{\circ} 44^{\prime} 00^{\prime \prime} \mathrm{S}$ & $77^{\circ} 09^{\prime} 00^{\prime \prime} \mathrm{O}$ \\
8 & Lima & Chancay & $11^{\circ} 34^{\prime} 07^{\prime \prime} \mathrm{S}$ & $77^{\circ} 16^{\prime} 11^{\prime \prime O}$ \\
9 & Arequipa & Arequipa & $17^{\circ} 01^{\prime} 11^{\prime \prime} \mathrm{S}$ & $72^{\circ} 00^{\prime} 58^{\prime \prime} \mathrm{O}$ \\
10 & Lima & Huacho & $01^{\circ} 06^{\prime} 24^{\prime \prime} \mathrm{S}$ & $77^{\circ} 36^{\prime} 18^{\prime \prime} \mathrm{O}$ \\
11 & Piura & Piura & $05^{\circ} 33^{\prime} 27^{\prime \prime} \mathrm{S}$ & $80^{\circ} 49^{\prime} 20^{\prime \prime} \mathrm{O}$ \\
\hline
\end{tabular}

fue evaluar los descriptores ecológicos de $A$. pacificus en los peces de interés comercial en la costa del Perú.

\section{Materiales y Métodos}

Se realizaron 49 muestreos en 11 zonas de muestreo de la costa peruana (Cuadro 1) en el periodo comprendido entre de marzo de 2016 y octubre de 2017. De estos. 31 muestreos se realizaron en 2016 y 18 muestreos en 2017.

Se colectaron 12017 pescados pertenecientes a 28 especies. De estos, 715 fueron en la Región Lima (Pucusana [134], Chorrillos [351], Ancón [85], Chancay [80], Huacho [65]) (Figura 1), 8251 en la Provincia Constitucional del Callao, 878 en Pisco, 1060 en Arequipa (1,060) (Figura 2), y 38 en Chimbote, 920 en Chiclayo y 155 en Piura (Figura 3).

Los procedimientos de parasitología clásica (colecta, fijación e identificación de los plerocercoides [PLs]) fueron realizados en los laboratorios de la Dirección de Investigación, Desarrollo, Innovación y Transferencia Tecnológica (DIDITT) del Instituto Tecnológico de la Producción (ITP) y en el laboratorio de Parasitología en Fauna Silvestre y Zoonosis de la Universidad Nacional Mayor de San Marcos (UNMSM), Lima, Perú.

Los pescados fueron evaluados sobre una mesa de disección. La búsqueda de quistes de PLs de A. pacificus se realizó en la superficie de las vísceras. Los PLs extraídos se lavaron en solución salina al $0.85 \%$ y se identificaron mediante estereoscopio (Olympus ${ }^{\circledR}$ ) y se fijaron con alcohol al $70 \%$. La prevalencia, abundancia e intensidad media de los PLs fueron determinados acorde con los criterios de Margolis et al. (1982), Bush et al. (1997) y Zander (2001), respectivamente. Las fórmulas empeladas fueron:

- $\quad$ Abundancia $=\mathrm{N} .^{\circ}$ de parásitos colectados / N..$^{\circ}$ de hospederos de la muestra

- $\quad$ Prevalencia $=\left(N .^{\circ}\right.$ de hospederos infestados / N. ${ }^{\circ}$ de hospederos examinados)*100 


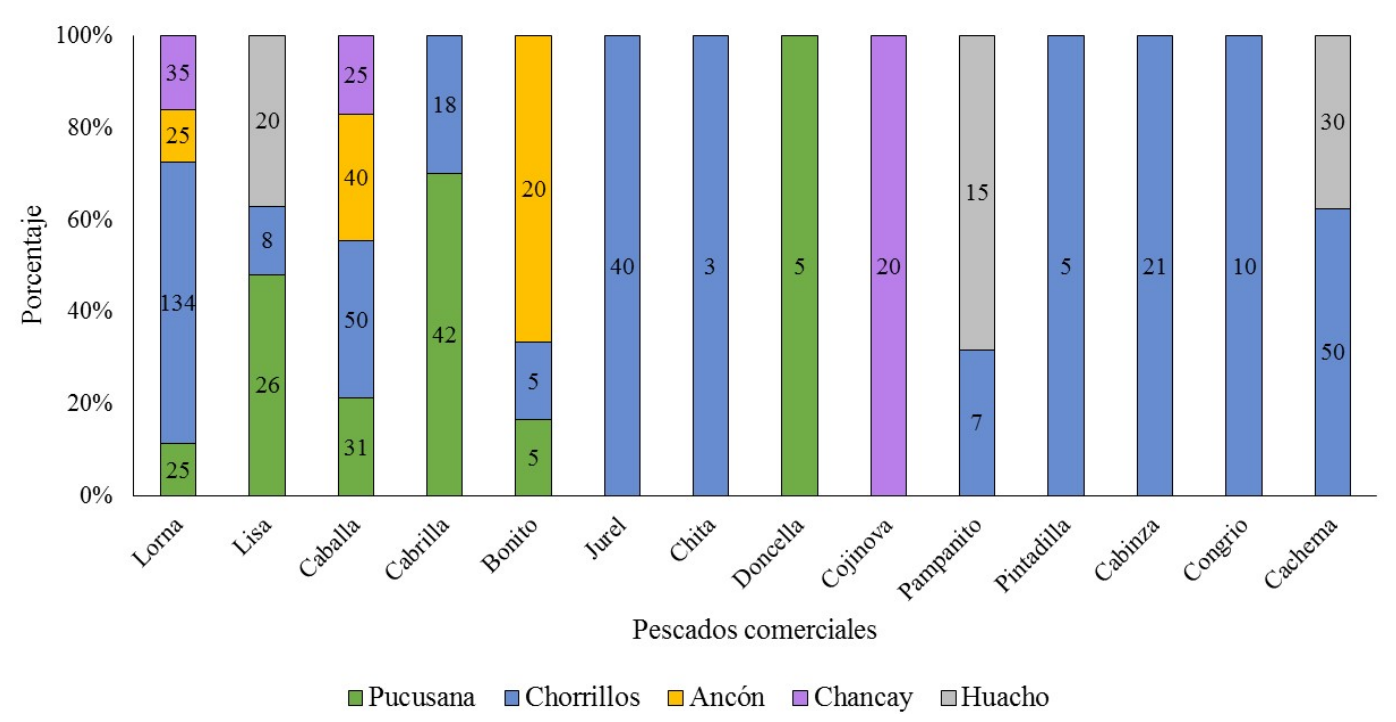

Figura 1.Especímenes colectados en la Región Lima (Pucusana, Chorrillos, Ancón, Chancay y Huacho): lorna (S. deliciosa), lisa (M. cephalus), caballa (S. japonicus), cabrilla ( $P$. humeralis), bonito (S. chiliensis), jurel (T. murphyi), chita (Anisotremus scapularis), doncella (Hemanthias peruanus), cojinova (Seriolella violacea), pampanito (Trachinotus paitensis), pintadilla (Cheilodactylus variegatus), cabinza (I. conceptionis), congrio (Genypterus maculatus) y cachema (Cynoscion analis)



Figura 2. Especímenes colectados en la Provincia Constitucional del Callao y en las provincias de Pisco y Arequipa: anchoveta (E. ringens), lorna (S. deliciosa), lisa (M.cephalus), caballa (S. japonicus), cabrilla (P. humeralis), bonito ( $S$. chiliensis), jurel ( $T$. murphyi), espejo (Selene peruviana), pejerrey (Odontesthes regia), chita ( $A$. scapularis), doncella (H. peruanus), cojinova ( $S$. violacea), pintadilla ( $C$. variegatus), ojo de uva (Hemilutjanus macrophthalmos), cabinza (I. conceptionis), pez volador (Cheilopogon heterurus), corcovado (Caranx lugubris), bobo (Joturus pichardi) y machete (Ethmidium maculatum). 


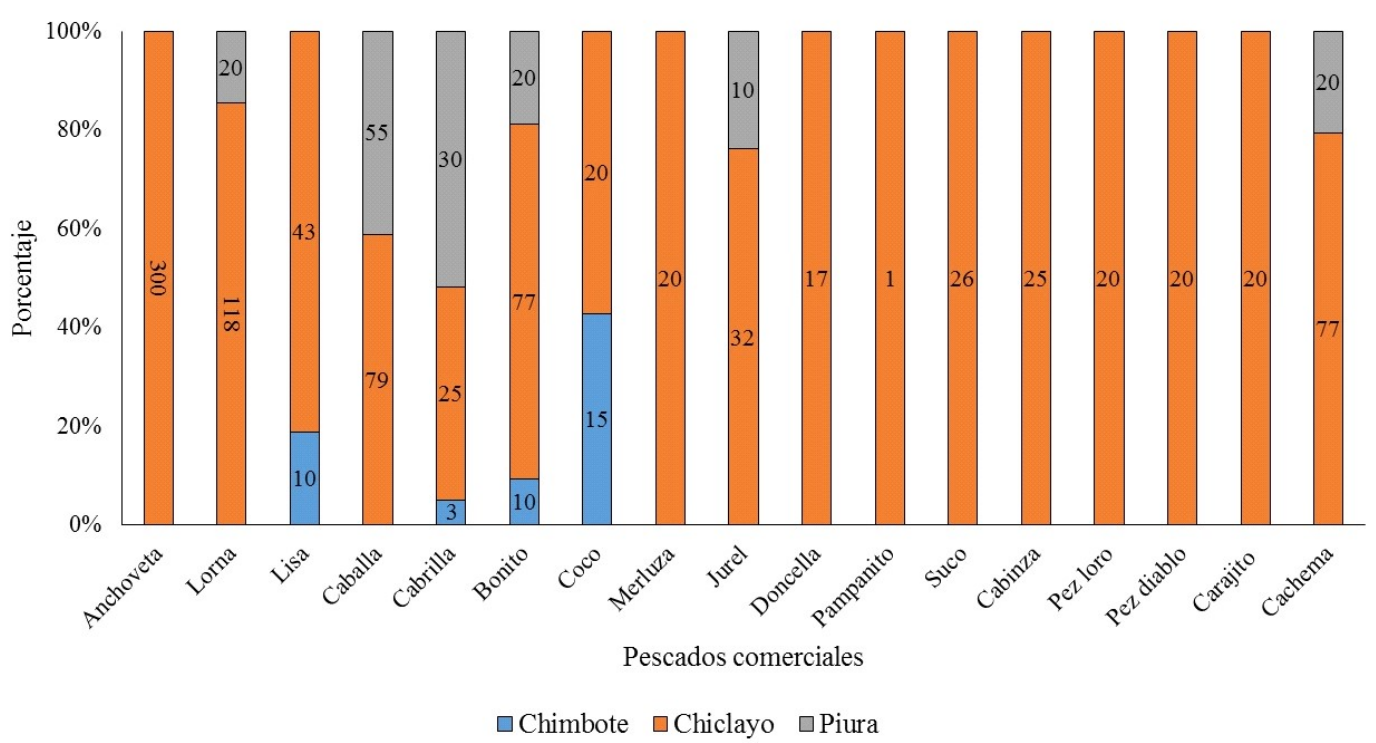

Figura 3. Especímenes colectados en las provincias de Chimbote, Chiclayo y Piura: anchoveta (E. ringens), lorna (S. deliciosa), lisa (M. cephalus), caballa (S. japonicus), cabrilla (P. humeralis), bonito (S. chiliensis), coco (Paralonchurus peruanus), merluza (M. gayi peruanus), jurel (T. murphyi), doncella (H. peruanus), pampanito (Trachinotus paitensis), cabinza (I. conceptionis), pez loro (Oplegnathus insignis), pez diablo (Scorpaena plumieri mystes), carajito (Diplectrum conceptione) y cachema ( $C$. analis)

- $\quad$ Intensidad media $=\left(\mathrm{N} .^{\circ}\right.$ de parásitos de una especie / N. ${ }^{\circ}$ de especies infestadas con el parásito)

\section{Resultados}

Los PLs encontrados presentaron las siguientes formas:

(A) Acorazonado: cuerpo cilíndrico, más o menos robusto, entre 6.5 y $7 \mathrm{~mm}$ de largo, superficie del cuerpo lisa, con 1 o 2 pequeñas constricciones en el cuerpo, color blanquecino, escólex grande, de borde anterior redondeado y botrias poco profundas (Figura 4a).

(B) Esférico: cuerpo cilíndrico, 12 a $13 \mathrm{~mm}$ de largo, color blanquecino, superficie del cuerpo con constricciones poco profun- das, escólex con borde anterior redondeado y con botrias profundas (Figura $4 b)$.

(C) Lanceolado: cuerpo cilíndrico, de superficie lisa, extremo anterior de forma triangular con el ápice redondeado. Posterior al escólex se presenta una dilatación del cuerpo, botrias grandes y profundas (Figura 4c).

Se encontraron 220 peces parasitados, 1o cual equivale al $2.04 \%$ de peces muestreados. Así mismo, se encontraron 515 parásitos. $S$. deliciosa fue la especie más parasitada con una prevalencia de $24.96 \%$ y presentó la mayor cantidad de PLs (448). Asimismo, E. ringens), M. cephalus, $S$. japonicus, $P$. humeralis y $I$. conceptionis presentaron prevalencias de $0.55,0.56,1.79$, 1.53 y $3.95 \%$, respectivamente (Cuadro 2 ). 

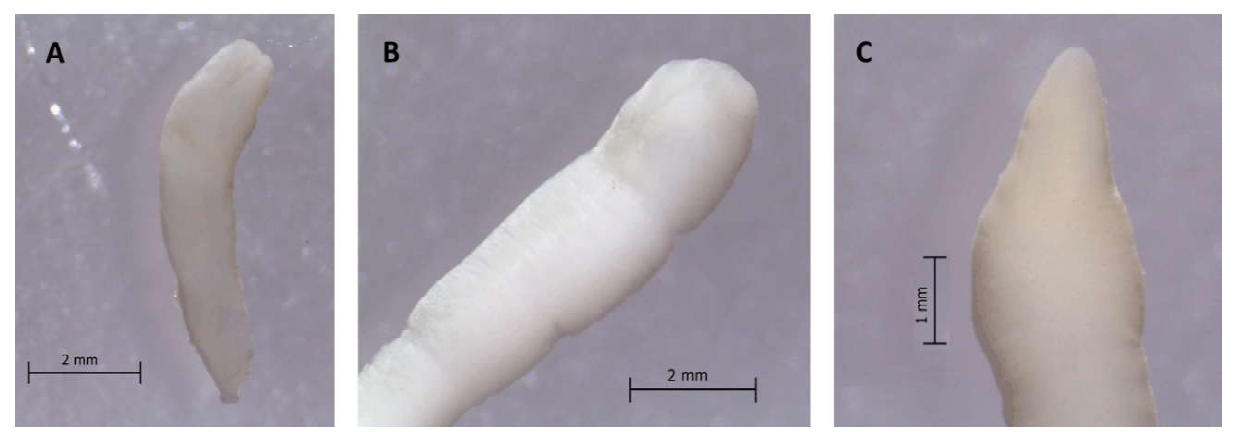

Figura 4. Formas de plerocercoides de Adenocephalus pacificus. A: Acorazonado, B: Esférico, C: Lanceolado

Cuadro 2. Descriptores parasitológicos de pescados muestreados en el litoral del Perú durante el periodo marzo de 2016 y octubre de 2017

\begin{tabular}{|c|c|c|c|c|c|c|}
\hline \multirow[t]{2}{*}{ Nombre } & \multicolumn{2}{|c|}{ Peces (n) } & \multirow{2}{*}{$\begin{array}{l}\text { Total de } \\
\text { PLs }^{1}\end{array}$} & \multirow{2}{*}{$\begin{array}{c}\text { Prevalencia } \\
(\%)\end{array}$} & \multirow{2}{*}{$\begin{array}{l}\text { Intensidad } \\
\text { media }\end{array}$} & \multirow{2}{*}{$\begin{array}{l}\text { Abun- } \\
\text { dancia }\end{array}$} \\
\hline & Muestreados & Infestados & & & & \\
\hline $\begin{array}{l}\text { Anchoveta } \\
\text { (E. ringens) }\end{array}$ & 9254 & 51 & 52 & 0.55 & 1.02 & 0.006 \\
\hline $\begin{array}{l}\text { Lorna } \\
\text { (S. deliciosa) }\end{array}$ & 617 & 154 & 448 & 24.96 & 2.91 & 0.726 \\
\hline $\begin{array}{l}\text { Lisa } \\
\text { (M. cephalus) }\end{array}$ & 179 & 1 & 1 & 0.56 & 1.00 & 0.006 \\
\hline $\begin{array}{l}\text { Caballa } \\
\text { (S. japonicus) }\end{array}$ & 446 & 8 & 8 & 1.79 & 1.00 & 0.018 \\
\hline $\begin{array}{l}\text { Cabrilla } \\
\text { (P. humeralis })\end{array}$ & 196 & 3 & 3 & 1.53 & 1.00 & 0.015 \\
\hline $\begin{array}{l}\text { Cabinza } \\
\text { (I. conceptionis) }\end{array}$ & 76 & 3 & 3 & 3.95 & 1.00 & 0.039 \\
\hline
\end{tabular}

${ }^{1}$ Plerocercoides

La abundancia parasitaria más baja se encontró en $M$. cephalus y E. ringens (0.006), mientras que la más alta se presentó en $S$. deliciosa (0.726). Con respecto a la carga parasitaria (presencia de PLs por hospedero), M. cephalus, S. japonicus, $P$. humeralis e $I$. conceptionis fueron infestadas con un PL. Así mismo, solo un espécimen de $E$. ringens presentó 2 PLs y $S$. deli- ciosa se encontró infestada con más de un PL. En algunos casos, esta infestación llego a 16 PLs en un solo hospedero (datos no presentados). Además, en E. ringens y $S$. deliciosa se presentaron las tres formas de PLs (A, B y C), en $S$. japonicus, P. humeralis e I. conceptionis se presentaron las formas A y B y en $M$. cephalus se presentó la forma B (Cuadro 3). 
Cuadro 3. Especies de peces infestados con quistes de plerocercoides (PLs) de Adenocephalus pacificus en la costa peruana

\begin{tabular}{ccccc}
\hline $\begin{array}{c}\text { Zona de } \\
\text { colecta }\end{array}$ & $\begin{array}{c}\text { Fecha de } \\
\text { colecta }\end{array}$ & Especie colectada & $\begin{array}{c}\mathrm{N}^{\mathrm{o}} \text { de } \\
\mathrm{PL}\end{array}$ & $\begin{array}{c}\text { Forma de } \\
\mathrm{PL}\end{array}$ \\
\hline Chorrillos & Oct-17 & Lorna (Sciaena deliciosa) & 22 & $\mathrm{~A} / \mathrm{B} / \mathrm{C}$ \\
& Ago-17 & Lorna (Sciaena deliciosa) & 71 & $\mathrm{~A} / \mathrm{B} / \mathrm{C}$ \\
Chiclayo & Oct-17 & Lorna (Sciaena deliciosa) & 219 & $\mathrm{~A} / \mathrm{B} / \mathrm{C}$ \\
& Oct-17 & Anchoveta (Engraulis ringens) & 12 & $\mathrm{~A} / \mathrm{B} / \mathrm{C}$ \\
& Jul-16 & Lorna (Sciaena deliciosa) & 52 & $\mathrm{~A} / \mathrm{B} / \mathrm{C}$ \\
& Mar-17 & Caballa (Scomber japonicus) & 3 & $\mathrm{~A} / \mathrm{B}$ \\
Callao & Mar-17 & Cabinza (Isacia conceptionis) & 3 & $\mathrm{~A} / \mathrm{B}$ \\
& Oct-17 & Lorna (Sciaena deliciosa) & 54 & $\mathrm{~A} / \mathrm{B} / \mathrm{C}$ \\
& Mar-16 & Anchoveta (Engraulis ringens) & 12 & $\mathrm{~A} / \mathrm{B} / \mathrm{C}$ \\
& Ago-16 & Anchoveta (Engraulis ringens) & 15 & $\mathrm{~A} / \mathrm{B} / \mathrm{C}$ \\
& Dic-16 & Anchoveta (Engraulis ringens) & 4 & $\mathrm{~A} / \mathrm{B} / \mathrm{C}$ \\
& Ene-17 & Anchoveta (Engraulis ringens) & 9 & $\mathrm{~A} / \mathrm{B} / \mathrm{C}$ \\
Arequipa & Mar-17 & Lorna (Sciaena deliciosa) & 7 & $\mathrm{~A} / \mathrm{B} / \mathrm{C}$ \\
& Jul-17 & Lorna (Sciaena deliciosa) & 9 & $\mathrm{~A} / \mathrm{B} / \mathrm{C}$ \\
Pisco & Nov-16 & Lorna (Sciaena deliciosa) & 6 & $\mathrm{~A} / \mathrm{B} / \mathrm{C}$ \\
Pucusana & Nov-16 & Cabrilla (Paralabrax humeralis) & 3 & $\mathrm{~A} / \mathrm{B}$ \\
& Mar-17 & Lorna (Sciaena deliciosa) & 2 & $\mathrm{~A} / \mathrm{B} / \mathrm{C}$ \\
& Abr-16 & Lorna (Sciaena deliciosa) & 6 & $\mathrm{~A} / \mathrm{B} / \mathrm{C}$ \\
& Abr-16 & Lisa (Mugil cephalus) & 1 & $\mathrm{~B}$ \\
& Abr-16 & Caballa (Scomber japonicus) & 5 & $\mathrm{~A} / \mathrm{B}$ \\
\hline
\end{tabular}

${ }^{1}$ A: Acorazonado, B: Esférico, C: Lanceolado

\section{Discusión}

La especie con mayor infestación fue la lorna (S. deliciosa) con una prevalencia de $24.96 \%$, siendo este reporte el más alto presentado hasta la fecha con respecto a esta especie en el Perú (Tantaleán, 1975; Llerena et al., 2001; Chero et al., 2014a; Chero, 2017). Además, es la prevalencia más alta en comparación con otras especies de peces, como el $23.30 \%$ en corvina (Cilus gilberti) encontrado por Chero et al. (2014c). Asimismo, la presencia de múltiples PLs, hasta 16 en un solo individuo y las tres formas de PLs en el mismo individuo, corresponde al primer reporte de esta particularidad en esta especie.
Este estudio es el primero en reportar la presencia de PLs de A. pacificus en lisa (M. cephalus), con una prevalencia de $0.56 \%$, dado que no ha sido encontrada en otros estudios donde se ha evaluado esta especie (Tantaleán, 1975; Iannacone y Alvariño, 2009b; Cajachagua et al., 2012; VásquezRuiz y Jara-Campos, 2012; Chero et al., 2014b; Serrano-Martínez et al., 2017). Así mismo, este sería el primer reporte de la presencia de PLs de $A$. pacificus en el país en cabinza (I. conceptionis) con una prevalencia de 3.95\%. Iannacone et al. (2015) hicieron un estudio en esta especie en una población de 123 individuos entre diciembre de 2010 y marzo de 2011, sin encontrar estos PLs, lo cual se podría deber a cambios en el ecosistema marino. 
El $1.79 \%$ de prevalencia de $A$. pacificus encontrada en caballa ( $S$. japonicus) fue similar al 1.7\% reportado por Oliva et al. (2008). Por otro lado, Cabrera y Tantaleán (1995) y Cruces et al. (2014) no encontraron este parásito en esta especie. El $1.53 \%$ de prevalencia en cabrilla ( $P$. humeralis) difiere del $3.5 \%$ reportado por Innacone y Alvariño (2009a), posiblemente debido al mayor tamaño de muestra $(n=369)$ en ese estudio. En forma similar, el $0.56 \%$ de prevalencia en anchovetas (E. ringens) fue menor del $1.34 \%$ señalado por Céspedes-Chombo et al. (2017), posiblemente debido a diferencias en el tamaño de muestra entre dicho estudio $(n=3420)$ y el presente trabajo $(n=9254)$ y las épocas de muestreo. No obstante, en ambos estudios se hallaron 2 PLs en un individuo.

Los resultados evidencian una baja prevalencia de infestación por larvas PLs de $A$. pacificus en los peces marinos de la costa peruana. Tantaleán (1993) argumenta que el aumento de la carga parasitaria de $A$. pacificus podría depender del fenómeno $\mathrm{El}$ Niño y de la estacionalidad (verano). Si bien hay estudios realizados en épocas de fenómeno El Niño (Pérez et al., 1999; Oliva et al., 2008; Iannacone y Alvariño, 2009a) con prevalencias menores que en años en los que no se presenta este fenómeno, también se dispone de estudios (Vasquez-Ruiz y JaraCampos, 2012; Iannacone et al., 2010, 2012, 2015; Payes, 2013) realizados en años con presencia del fenómeno El Niño donde no se presentaron casos de A. pacificus. Esto podría ser debido a que estos estudios se realizaron en terminales pesqueros y no se conoce con exactitud la procedencia de los individuos y a la calidad de almacenamiento de estos. En el presente estudio, en 2016 (sin fenómeno El Niño) se reportó 104 PLs y en el 2017 (con fenómeno El Niño) se reportó 411 PLs, resultado que está acorde con lo reportado por Tantaleán (1993). No obstante, cabe señalar que el número de PLs por año no considera la estación ni huésped; en tanto que en este estudio, se puede ver en el Cuadro 3, que el número de PLs en verano es menor que en las estaciones de invierno en ambos años y en primavera en 2017.
Desde el punto de vista epidemiológico, este estudio aporta nuevos datos debido a que se evaluaron especies de peces que a la fecha no se habían estudiado. Entre estos, bobo (Joturus pichardi), carajito (Diplectrum conceptione), corcovado (Caranx lugubris), doncella (Hemanthias peruanus), espejo (Selene peruviana), ojo de uva (Hemilutjanus macrophthalmos), pejerrey (Odontesthes regia), pez diablo (Scorpaena plumieri mystes), pez loro (Oplegnathus insignis) y pez volador (Cheilopogon heterurus) son especies que no presentaron PLs de A. pacificus.

Se concluye que en los periodos del fenómeno El Niño es muy probable que la prevalencia de $A$. pacificus aumente en comparación con los años en los que no se presenta; pero en el presente estudio se ha demostrado que la estacionalidad no es relevante, y debe haber otras variables que estén influenciando en esta manifestación. Es así que se deben definir variables adicionales para determinar la particularidad que se expresa en años del Fenómeno El Niño. Por otro lado, es probable que haya infestación por más de un PL por hospedero, así como la presencia en el mismo hospedero de las tres formas de PLs reportadas en este estudio. Todos los PLs encontrados en este estudio pertenecen a A. pacificus. Es importante indicar, además, que se han hecho estudios moleculares de PLs obtenidos de peces marinos (colectados en este estudio) de la costa peruana, así como de adultos procedentes de humanos y lobos marinos (Mondragón, 2017; Marroquín, 2018; Londoñe et al., 2017a,b; 2018) que demuestran que los PLs corresponden a la especie $A$. pacificus.

\section{Agradecimientos}

Se agradece al Instituto Tecnológico de la Producción por haber brindado las facilidades para utilizar sus laboratorios, a la Dra. Rosa Martínez Rojas del Laboratorio de Parasitología en Fauna Silvestre y Zoonosis de la Universidad Nacional Mayor de San Marcos (UNMSM), por su apoyo en el Pro- 
yecto «Identificación molecular de especies de Diphyllobothrium en peces de mayor índice de consumo en el Perú», Convenio $\mathrm{N}^{\circ}$ 215-2015-FONDECYT. También se agradece al Dr. Manuel Tantaleán por la identificación de los plerocercoides estableciendo sus características morfológicas. Este trabajo fue subvencionado por el Fondo Nacional de Desarrollo Científico, Tecnológico y de Innovación Tecnológica (FONDECYT), en el marco del Convenio $\mathrm{N}^{\circ}$ 215-2015FONDECYT.

\section{Literatura Citada}

1. Berger S. 2017. Taenia and Diphyllobothrium: global status. Los Ángeles, CA: Gideon Informatics. $78 \mathrm{p}$.

2. Bush AO, Lafferty KD, Lotz JM, Shostak $A W$. 1997. Parasitology meets ecology on its own terms: Margolis et al. revisited. J Parasitol 83: 575-583. doi: $10.2307 / 3284227$

3. Cabrera R, Tantaleán M. 1995. Algunos helmintos de Scomber japonicus «caballa» de la Reserva Nacional de Paracas. Biotempo 2: 85-86. doi: 10.31381/biotempo.v2i0.1541

4. Cajachagua C, Dueñas B, Ricalde J, Villanueva F, Iannacone J. 2012. Índices de agregación de la parasitofauna de Sciaena deliciosa (Osteichthyes: Sciaenidae) y Mugil cephalus (Osteichthyes: Mugilidae) del terminal pesquero de chorrillos, Lima, Perú, 2012. Biologist 10: 33 .

5. Céspedes-Chombo R, Díaz-Pereyra K, Salas-Maldonado A, Iannacone J. 2017. Descriptores ecológicos parasitarios de Adenocephalus pacificus (Nybelin, 1931) (Cestoda: Diphyllobothriidae) en Engraulis ringens (Jenyns, 1842) «Anchoveta» (Clupeiformes, Engraulidae) en la Costa del Perú. Neotrop Helminthol 11: 395-404.

6. Chero J, Cruces C, Iannacone J, Sáez G, Alvariño L, Rodríguez C, Rodríguez $\mathrm{H}$, et al. 2014a. Índices parasitológicos de la merluza peruana Merluccius gayi peruanus (Ginsburg, 1954) (Perciformes: Merlucciidae) adquiridos del terminal pesquero de Ventanilla, Callao, Perú. Neotrop Helminthol 8: 131-140.

7. Chero J, Iannacone J, Cruces C, Sáez G, Alvariño L. 2014b. Comunidad de metazoos parásitos de la corvina Cilus gilberti (abbott, 1899) (Perciformes: Sciaenidae) en la zona costera de Chorrillos, Lima, Perú. Neotrop Helminthol 8: 163-182.

8. Chero J, Sáez G, Iannacone J, Aquino W. 2014c. Aspectos ecológicos de los helmintos parásitos de lorna Sciaena deliciosa (Tschudi, 1846) (Perciformes: Sciaenidae) adquiridos del terminal pesquero de Ventanilla, Callao, Perú. Neotrop Helminthol 8: 59-76.

9. Chero J, Sáez G, Iannacone J, Cruces C, Alvariño L, Luque J. 2016. Ecología comunitaria de metazoos parásitos del bonito Sarda chiliensis Cuvier, 1832 (Perciformes: Scombridae) de la costa peruana. Rev Inv Vet Perú 27: 539555. doi: 10.15381/rivep.v27i3.12008

10. Chero J. 2017. Biodiversidad de metazoos parásitos en peces de la zona marino-costera de Lima, Perú. Tesis de Magíster. Lima: Univ. Nacional Mayor de San Marcos. 101 p.

11. Cruces C, Chero J, Iannacone J, Diestro A, Sáez G, Alvariño L. 2014. Metazoos parásitos de «caballa» Scomber japonicus Houttuyn, 1782 (Perciformes: Scombridae), del puerto de Chicama, La Libertad, Perú. Neotrop Helminthol 8: 357-381.

12. Hernández-Orts JS, Scholz, T, Brabec J, Kuzmina T, Kuchta R. 2015. High morphological plasticity and global geographical distribution of the Pacific broad tapeworm Adenocephalus pacificus (syn. Diphyllobothrium pacificum): molecular and morphological survey. Acta Tropica 149: 168-178. doi: 10.1016/j.actatropica.2015.05.017 
13. Iannacone J, Alvariño L, Chero J, Sáez G 2015. Comunidad parasitaria de cabinza Isacia conceptionis (Cuvier y Valenciennes, 1830) (Perciformes: Haemulidae) en la zona de Chorrillos, Lima, Perú. Rev Inv Vet Perú 26: 96110. doi: 10.15381/rivep.v26i1.10943

14. Iannacone J, Alvariño L. 2009a. Dinámica poblacional de la diversidad parasitaria de la «cabrilla» Paralabrax humeralis (Teleostei: Serranidae) en Chorrillos, Lima, Perú. Neotrop Helminthol 3: 73-88.

15. Iannacone J, Alvariño L. 2009 b. Metazoos parásitos de Mugil cephalus Linnaeus, 1758 (Mugilidae: Perciformes) procedentes del terminal pesquero de Chorrillos, Lima, Perú. Neotrop Helminthol 3: 15-28.

16. Iannacone J, Cárdenas-Callirgo J, Alvariño L. 2010. Ecología comunitaria de los parásitos de la chilindrina Stromateus stellatus (Cuvier, 1829) (Perciformes: Stromateidae) de la zona costera de Chorrillos, Lima, Perú. Neotrop Helminthol 4: 159-167.

17. Iannacone J, Sánchez V, Olazábal N, Salvador C, Alvariño L, Molano J. 2012. Índices ecológicos de los parásitos de Scartichthys gigas (Steindachner, 1876) (Perciformes: Blenniidae) de las costas de Lima, Perú. Neotrop Helminthol 6: 191-203.

18. Kuchta R, Esteban JG, Brabec J, Scholz T. 2014. Misidentification of Diphyllobothrium species related to global fish trade, Europe. Emerg Infect Dis 20: 1955-1957. doi: 10.3201/ eid2011.140996

19. Kuchta R, Serrano-Martínez $M$, Scholz T. 2015. Pacific broad taperworm Adenocephalus pacificus as a causative agent of globally reemerging Diphyllobothriosis. Emerg Infect Dis 21: 1697-1703. doi: 10.3201/eid2110.150516

20. Llerena C, Chávez A, Casas E. 2001. Frecuencia de larvas Diphyllobothriidae y larvas Anisakidae en peces marinos comerciales del terminal pesquero de Ventanilla - Callao. Rev Inv Vet Perú 12: 5862. doi: 10.15381/rivep.v12i1.7425
21. Londoñe P, Tantaleán M, Martínez $R$, García E, Díaz K, Céspedes $R$, Sánchez C. 2017a. Identificación molecular de Adenocephalus pacificus (sin. Diphyllobothrium pacificum) en lorna (Sciaena deliciosa) y cabrilla (Diplectrum conceptione) capturadas en el litoral peruano. En: IX Congreso Cubano de Microbiología y Parasitología. La Habana, Cuba.

22. Londoñe P, Tantaleán M, García E, Sánchez C, Céspedes R, Díaz K. 2017b. Primera identificación molecular de Adenocephalus pacificus en anchoveta (Engraulis ringens) capturada en el litoral peruano. En: IX Congreso Cubano de Microbiología y Parasitología. La Habana, Cuba.

23. Londoñe P, Tantaleán M, SanchezRobinet C, Radulovici A, Borisenko A. 2018. A DNA barcode for Adenocephalus pacificus from Peru. In: $28^{\text {th }}$ ECCMID. Madrid-España: European Congress of Clinical Microbiology and Infectious Diseases.

24. Margolis L, Esch GW, Holmes JC, Kuris AM, Schad GA. 1982. The use of ecological terms in parasitology (Report of an ad hoc committee of The American Society of Parasitologists). J Parasitol 68:131-133. doi: 10.2307/ 3281335

25. Marroquín E. 2018. Identificación molecular de larvas plerocercoides del género Diphyllobothrium sp obtenidas en peces marinos de mayor consumo utilizando los marcadores moleculares ITS y 18S. Tesis de Biólogo Microbiólogo Parasitólogo. Lima: Univ. Nacional Mayor de San Marcos. 51 p.

26. Mondragón A. 2017. Identificación molecular de los estadios de plerocercoide y adulto de Diphyllobothrium sp obtenidos de humanos, lobos marinos y peces. Tesis de Biología. Lima: Univ. Ricardo Palma. $72 \mathrm{p}$.

27. Moore CV, Thompson RC, Jabbar A, Williams J, Rasiah K, Pallant L, Koehler AP, et al. 2017. Rare human infection with pacific broad tapeworm 
Adenocephalus pacificus, Australia. Emerg Infect Dis 22: 1510-1511. doi: 10.3201/eid2208.160156

28. Oliva M, Valdivia I, Costa G, Freitas $N$, Pinheiro De Carvalho M, Sánchez L, et al. 2008. What can metazoan parasites reveal about the taxonomy of Scomber japonicus Houttuyn in the coast of South America and Madeira Islands? J Fish Biol 72: 545-554. doi: 10.1111/j.1095-8649.2007.01725.x

29. Payes C. 2013. Prevalencia de parásitos en Sciaena deliciosa «lorna» (Tschudi, 1846) comercializados en los mercados del distrito de Tacna. Tesis de Biología. Tacna, Perú: Univ. Nacional Jorge Basadre Grohmann. 52 p.

30. Pérez I, Chávez A, Casas E. 1999. Presencia de formas parasitarias en peces comerciales del mar peruano. Rev Inv Vet Perú 10: 34-38.

31. [PRODUCE] Ministerio de la Producción. 2016. Anuario estadístico pesquero y acuícola. 2016. Ministerio de la Producción. La actividad productiva del sector en números. Perú. PRODUCE. $204 \mathrm{p}$.

32. [PRODUCE] Ministerio de la Producción. 2017. Boletín del Sector Pesquero 2017. Ministerio de la Producción. Desenvolvimiento productivo de la actividad pesquera. Perú. PRODUCE. $46 \mathrm{p}$.

33. Sanchez M, Knoff M, Corrêa-Gomes D, Brener B. 2016. Checklist of Platyhelminth parasites of humans deposited in Helminthological Collection of the Oswaldo Cruz Institute, Brazil. Neotropic Helminthol 10: 73-84.
34. Scholz, T, Kuchta R. 2016. Fish-borne, zoonotic cestodes (Diphyllobothrium and relatives) in cold climates: a neverending story of neglected and (re)emergent parasites. Food Waterborne Parasitol 4: 23-38. doi: 10.1016/ j.fawpar.2016.07.002

35. Serrano-Martínez E, Quispe HM, Hinostroza ME, Plasencia PL. 2017. Detección de parásitos en peces marinos destinados al consumo humano en Lima Metropolitana. Rev Inv Vet Perú 28: 160-168. doi: 10.15381/rivep.v28i1.12935

36. Shimokawa C. 2013. Estudio sobre viabilidad de consorcios de exportacioìn en Pesca. Lima, Peruì. 59 p.

37. Tantaleán M, Huiza A. 1994. Sinopsis de los parásitos de peces marinos de la costa peruana. Biotempo 1: 53-101.

38. Tantaleán M. 1975. Hallazgo de larvas plerocercoides de Diphyllobothriidae Lühe, 1910 (Cestoda) en peces del mar peruano. Bol Chil Parasitol 30: 18-20.

39. Tantaleán M. 1993. Algunos helmintos de mamíferos marinos del Perú y su importancia médica. Rev Per Med Trop UNMSM 7: 67-71.

40. Vásquez-Ruiz C, Jara-Campos C. 2012. Prevalencia e intensidad parasitaria en Coryphaena hippurus y Mugil cephalus (Teleostei) desembarcados en los puertos Salaverry y Paita (Perú). Sciendo 15: 22-32.

41. Zander CD. 2001. The guild as a concept and a means in ecological parasitology. Parasitol Res 87: 484-488. doi: $10.1007 / \mathrm{s} 004360100386$ 